\title{
Reduction of Chemical Noise in Electrospray Ionization Mass Spectrometry by Supplemental IR Activation
}

\author{
Myles W. Gardner and Jennifer S. Brodbelt \\ Department of Chemistry and Biochemistry, The University of Texas at Austin, Austin, Texas, USA
}

\begin{abstract}
Supplemental infrared (IR) activation was applied to reduce background chemical noise and increase analyte ion signal in a linear ion trap mass spectrometer. Peptides, proteins, and small molecules were all introduced by electrospray ionization, and when regions of chemical noise were isolated and subjected to IR irradiation, protonated analyte molecules were observed in the product ion mass spectra. By isolating the entire mass range (e.g., $\mathrm{m} / \mathrm{z} 400-2000)$ and then irradiating all ions in the trap, supplemental IR activation increased the signal of singly protonated peptides by almost $70 \%$ and by $40 \%-55 \%$ for the lower charge states of cytochrome $c$. This increase in analyte ion signal was less dramatic for the higher charge states of peptides and proteins. The chemical noise present in the mass spectra is attributed to incomplete desolvation of the electrospray, as the abundance of the protonated peptides observed upon supplemental IR activation of the chemical noise decreased with higher inlet capillary temperatures. Collision activation was not as effective for desolvating the ions present in the chemical noise. (J Am Soc Mass Spectrom 2009, 20, 2206-2210) @ 2009 American Society for Mass Spectrometry
\end{abstract}

$\mathrm{F}$ or successful implementation of electrospray ionization (ESI), desolvation of the analyte ions is necessary, and many methods have been developed to afford efficient desolvation, including the use of drying gases [1-3], collisional activation [2, 3], and heated inlet capillaries [4]. These techniques are applied to not only aid in desolvation but also to reduce the chemical noise in ESI mass spectra, which is typically saturated with solvated ion clusters. Other methods have been used to reduce the chemical noise that is due to specific contaminants, including using heated shield interfaces [5] and ion-molecule reactions to "filter" selected ions of interest [6,7]. To further eliminate chemical noise due to solvent clusters in quadrupole ion traps (QITs), broadband waveforms have been applied to gently collisionally activate and dissociate these solvated ions to convert them into free buffer-related ions for subsequent resonance ejection [8]. The nature of the chemical noise background in matrix-assisted laser desorption/ionization (MALDI) has been investigated in some detail and has also been determined to be in part due to matrix and analyte-matrix clusters [9, 10]; in fact, MALDI-TOF-TOF experiments on chemical noise ions of $\mathrm{m} / \mathrm{z}$ greater than that of the analyte ions yielded free analyte ions due to decay and desolvation of analyte-matrix clusters in the flight tube [10]. In ESI mass spectra, background noise is often observed at

Address reprint requests to Dr. J. S. Brodbelt, Department of Chemistry and Biochemistry, The University of Texas at Austin, 1 University Station A5300, Austin, TX 78712-0165, USA. E-mail: jbrodbelt@mail.utexas.edu virtually all $\mathrm{m} / \mathrm{z}$ values, and the source of this noise cannot often be determined [11]. While using "soft" ESI conditions (e.g., low inlet capillary temperatures and small potential differences between the capillary and skimmer) enhances the production of very stable hydrated peptide ions with upwards of 50 water adducts, which have been extensively studied by the Williams group [12,13], in this work we are investigating chemical noise in ESI mass spectra in which desolvating conditions are employed. Here, we report on utilizing supplemental IR activation in a linear ion trap mass spectrometer to reduce the chemical noise background observed in ESI mass spectra. Rather than focusing on eliminating background ions of specific $m / z$, we have applied IRMPD to activate ions of unresolved $\mathrm{m} / \mathrm{z}$ observed in noise regions of the ESI mass spectra. Upon IR irradiation of noise regions, analyte ions of high abundance are detected.

\section{Experimental}

\section{Mass Spectrometry and Materials}

All experiments were conducted on a modified LTQ XL linear ion trap mass spectrometer (Thermo Fisher Scientific, San Jose, CA) with the standard ESI source, which is directed at an angle to the inlet heated capillary. A ZnSe optical window was mounted to the back plate of the vacuum manifold on axis with the linear ion trap to transmit IR irradiation as previously described [14]. Supplemental IR activation was performed with a 
model 48-5 Synrad 50-W continuous wave $\mathrm{CO}_{2}$ laser (Mukilteo, WA), which emits radiation at $10.6 \mu \mathrm{m}$. For IR activation experiments, the irradiation time (0-50 $\mathrm{ms})$ and power $(25-50 \mathrm{~W})$ was varied. Wide-range isolation and IR activation experiments were conducted by turning off the isolation waveform through the diagnostics panel of the LTQTune v2.5.0 software. The laser was triggered through the LTQTune software only during the activation step in the scan function and not during ion accumulation, ion cooling, or ion transmission. (It should be noted that the laser beam does interact with all ions along the optical path from the linear ion trap to the skimmer cone but the laser beam does not pass through the inlet heated capillary, which is off-axis from the skimmer cone.) The automatic gain control was turned off for all experiments, and peak areas calculations were performed in triplicate. All samples were prepared at $10 \mu \mathrm{M}$ in 50:50:1 $\mathrm{H}_{2} \mathrm{O}$ / $\mathrm{MeOH} / \mathrm{HOAc}$ (vol/vol/vol), unless otherwise noted, and infused at $3 \mu \mathrm{L} / \mathrm{min}$ through a fused silica capillary (194 $\mu \mathrm{m}$ o.d., $98 \mu \mathrm{m}$ i.d.). The heated capillary temperature was maintained at $180{ }^{\circ} \mathrm{C}$ unless otherwise noted. The nitrogen sheath gas flow was set to 20 (arbitrary units, corresponding to $\sim 0.3 \mathrm{~L} / \mathrm{min}$ ) and no auxiliary gas flow was used. The peptides bradykinin (1059.5 Da), angiotensin I (1295.6 Da), fibrinopeptide A (1535.7 $\mathrm{Da})$, the protein cytochrome $c$, and reserpine (608.3 Da) were all obtained from Sigma-Aldrich (St. Louis, MO). All solvents and other chemicals were from Fisher Scientific (Fairlawn, NJ).

\section{Results and Discussion}

In a previous study in our lab, we employed infrared multiphoton dissociation (IRMPD) to differentiate and identify chromogenic cross-linked peptides from other non-cross-linked products [14]. It was observed that the abundances of some of the unmodified peptide cations actually increased upon IR irradiation, particularly when comparing peak areas in the wide-range isolation and IRMPD mass spectra. In a second time-resolved IRMPD study of peptides, it was also noted that ion abundances of selected precursor ions increased upon exposure to short irradiation times prior to observing ion dissociation upon exposure to longer irradiation times [15]. These increases in ion abundances upon IR irradiation were initially attributed to fluctuations in the ion current between the isolation and the IRMPD scans; however, as this phenomenon was consistently observed, other possible reasons for the increase in ion abundance were investigated.

For the present study, peptide samples were introduced by ESI using typical parameters to promote efficient ion desolvation; however, rather than isolating and activating a specific peptide ion, noise regions above the peptide ions or wide $\mathrm{m} / \mathrm{z}$ ranges (including the peptide ions) were subjected to IR irradiation. Typical wide range isolation and supplemental IR activation mass spectra of bradykinin are shown in Figure $1 \mathrm{a}$ and $\mathrm{b}$, respectively. The wide range isolation mass

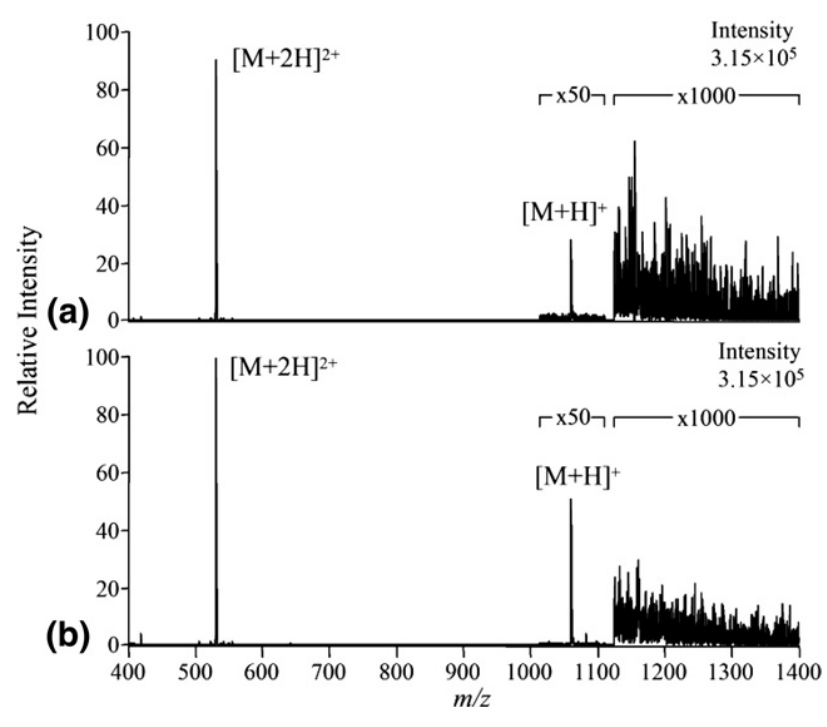

Figure 1. (a) Wide range isolation mass spectrum of $\mathrm{m} / \mathrm{z} 400-$ 2000 (30 ms) and (b) supplemental IR activation mass spectrum (30 ms, $25 \mathrm{~W}$ ) of bradykinin (M). Both spectra are normalized to the same intensity.

spectrum is identical to the conventional ESI mass spectrum of bradykinin (Figure 1a), and the range from $\mathrm{m} / \mathrm{z} 1000$ to 1100 is amplified to showcase the abundance of the protonated peptide. Upon $30 \mathrm{~ms}$ of supplemental IR irradiation at $25 \mathrm{~W}$, a significant increase in the abundance of singly protonated bradykinin is observed (Figure 1b). The peak area of $[\mathrm{M}+\mathrm{H}]^{+}$ increased by $68 \% \pm 17 \%$ ( $\sim 2260$ to $\sim 3800$ counts $)$, while the abundance of doubly-charged bradykinin increased by $3.8 \% \pm 2.0 \%$ ( $\sim 232000$ to $\sim 241000$ counts $)$. The total ion current (TIC) in the IR activation mass spectra was on average $4.5 \% \pm 1.2 \%(\sim 550,000$ to $\sim 575,000$ counts) greater than that measured in the wide range isolation mass spectra. These increases in peptide ion abundances parallel the decrease in the chemical noise, which is presumed to consist primarily of loosely bound solvated peptide ions of unresolved $\mathrm{m} / \mathrm{z}$. In the amplified region of $\mathrm{m} / \mathrm{z} 1300-1400$, there is a distinct difference in the noise level between the wide range isolation mass spectrum and the IRMPD mass spectrum (Figure 1a and $b$ ).

Similar increases in the abundances of the lower charge states of cytochrome $c$ were observed upon supplemental IR irradiation as shown in the wide-range isolation and IR activation mass spectra in Figure 2a and $b$. The abundances of the $8+$ through $10+$ charge states of cytochrome $c$ increased by $39 \%-55 \%$ upon IR irradiation. The peak areas of the $13+$ through $15+$ charge states only exhibited increases of $\sim 10 \%$, and the peak areas of the more highly charged cytochrome $c$ ions changed by less than $5 \%$. IR irradiation also effectively decreased the noise level above $\mathrm{m} / \mathrm{z} 1600$. The TIC increased by $\sim 15 \%$ between the isolation and the IR activation mass spectra, and this could be due to dissociation of highly solvated ions that have $\mathrm{m} / \mathrm{z}$ values that exceed the detectable mass range of the linear ion 


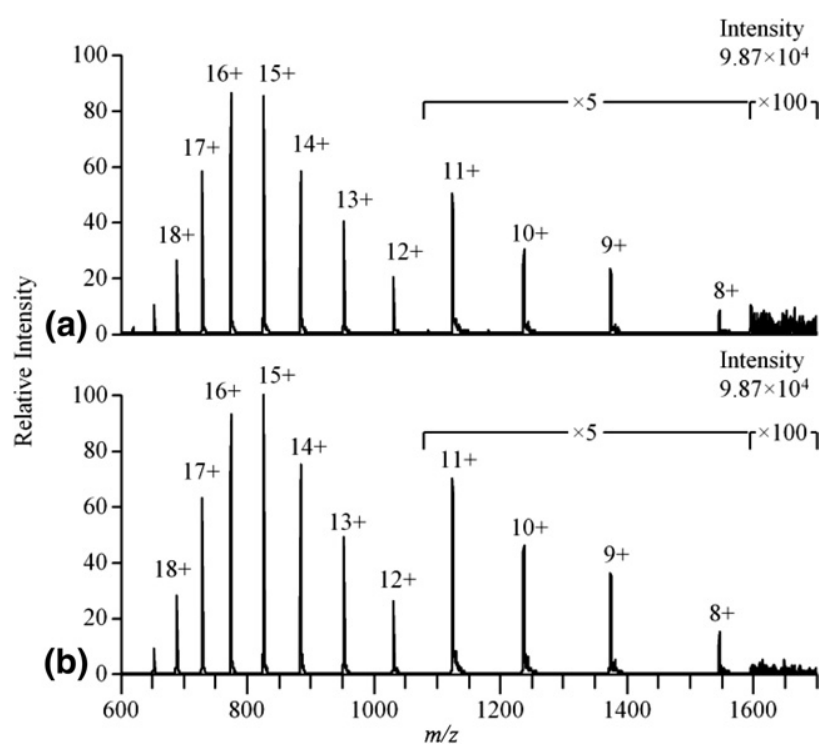

Figure 2. (a) Wide range isolation mass spectrum of $\mathrm{m} / \mathrm{z} 600-$ $2000(50 \mathrm{~ms})$ and (b) supplemental IR activation mass spectrum ( $50 \mathrm{~ms}, 50 \mathrm{~W}$ ) of cytochrome $c$. Both spectra are normalized to the same intensity.

trap (maximum $\mathrm{m} / \mathrm{z}$ of 2000) yet still have stable trajectories in the mass analyzer.

The effectiveness of IR activation to dissociate these solvated ions was also compared with collisional activation. The wide-range isolation mass spectrum of angiotensin I is shown in Figure 3a, in which ions of $\mathrm{m} / \mathrm{z}$ 1400-2000 were isolated. Upon IR irradiation, the entire noise region was drastically reduced in intensity, and protonated angiotensin in the $1+, 2+$, and $3+$ charge states were observed (Figure $3 b$ ). In fact, the abundances of the peptide ions were sufficiently high to allow subsequent collision induced dissociation (CID) $\mathrm{MS}^{3}$ experiments to confirm their identity (data not shown). As shown in Figure 3c, collisional activation of the range from $\mathrm{m} / \mathrm{z} 1450$ to 1550 (centered on $\mathrm{m} / \mathrm{z} 1500$ with an activation window of $100 \mathrm{u}$ ) also resulted in a decrease in the chemical noise of ions in resonance with the auxiliary collisional activation waveform, but high levels of noise remained above $\mathrm{m} / \mathrm{z}$ 1575. (Collisional activation waveforms could only be applied to activate ions within a mass window of $100 \mathrm{u}$ due to limitations of the instrument software.) Collisional activation of $\mathrm{m} / \mathrm{z} 1450-1550$ produced low abundances of peptide ions but at signal levels $\sim 30 \times$ lower in intensity than obtained by the IR activation process. As a more direct comparison between the effects of IR irradiation and collisional activation of specific noise regions, the narrow region from $\mathrm{m} / \mathrm{z} 1900$ to 2000 was isolated after infusion of a solution containing bradykinin (Supplemental Figure $1 \mathrm{a}$, which can be found in the electronic version of this article). and either subsequently irradiated or subjected to collisional activation. IR irradiation yielded an intense signal of singly protonated bradykinin $(m / z$ 1060.3) while collisional activation produced the same peptide ions at a $150 \times$ lower intensity (Supplemental Figure $1 b$ and $c$ ).
The temperature of the inlet heated capillary was varied to provide more evidence that the source of chemical noise in the ESI mass spectra was solvated ions of unresolved $\mathrm{m} / \mathrm{z}$. For a solution containing reserpine, the $m / z$ region 1900-2000 was isolated and subjected to $30 \mathrm{~ms}$ of IR irradiation to yield protonated reserpine $(\mathrm{m} / \mathrm{z} 609.7)$, and the peak area of this ion was plotted versus heated capillary temperature (Figure 4). As a control, the peak area of protonated reserpine in the ESI mass spectra without isolation or IR activation was also plotted. At low capillary temperatures, IR activation of $\mathrm{m} / \mathrm{z} 1900-2000$ at $120^{\circ} \mathrm{C}$ yielded the highest abundance of protonated reserpine, corresponding to significant IR desolvation of a large population of solvated ions. As the capillary temperature was increased, the abundance of protonated reserpine produced upon IR activation decreased, in accordance with the existence of a smaller population of solvated ions due to more efficient desolvation of the ions exiting the ESI capillary. In the absence of IR activation, the abundance of protonated reserpine in the ESI mass spectra increased with higher inlet capillary temperatures, indicative of the disruption of more of the solvent clusters and their conversion into desolvated reserpine.

The abundance of various bradykinin peptide ions produced upon IR activation was also monitored as a function of the $\mathrm{m} / \mathrm{z}$ window isolated and irradiated, ranging from $m / z$ 1100-1200 to $m / z$ 1900-2000. IR activation of the $m / z$ region just above that of protonated bradykinin $(m / z$ 1060.3) yielded the highest abundances of

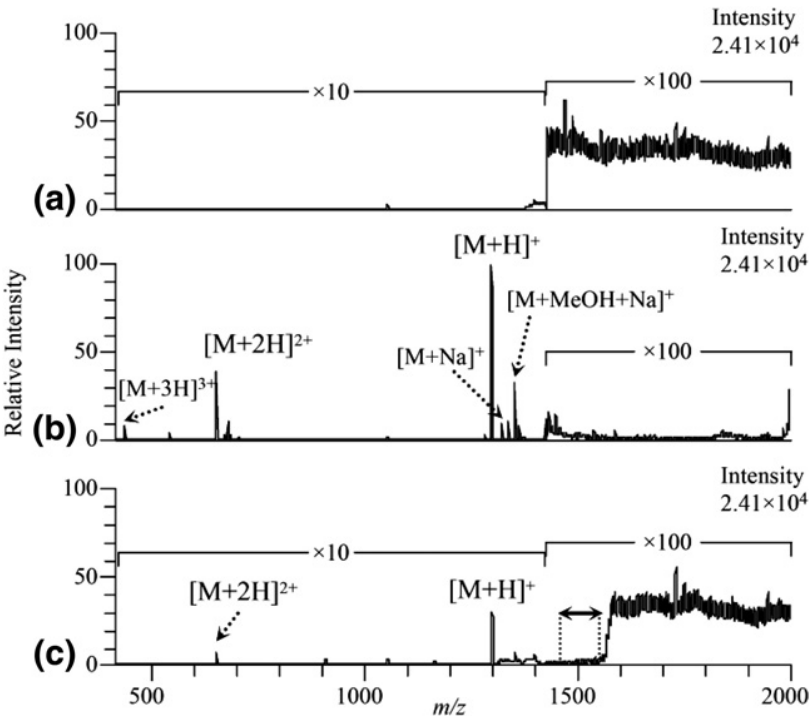

Figure 3. (a) Wide range isolation mass spectrum of $\mathrm{m} / \mathrm{z} 1400$ 2000 (30 ms), (b) supplemental IR activation mass spectrum (30 $\mathrm{ms}, 50 \mathrm{~W})$, and (c) collisional activation mass spectrum $(30 \mathrm{~ms}$, $100 \%$ normalized collision energy) of angiotensin I (M). Only ions of $m / z$ 1450-1550 were activated in the collisional activation mass spectrum (range indicated with double-headed arrow) while all ions of $m / z$ 1400-2000 were isolated. All spectra are normalized to the same intensity. 


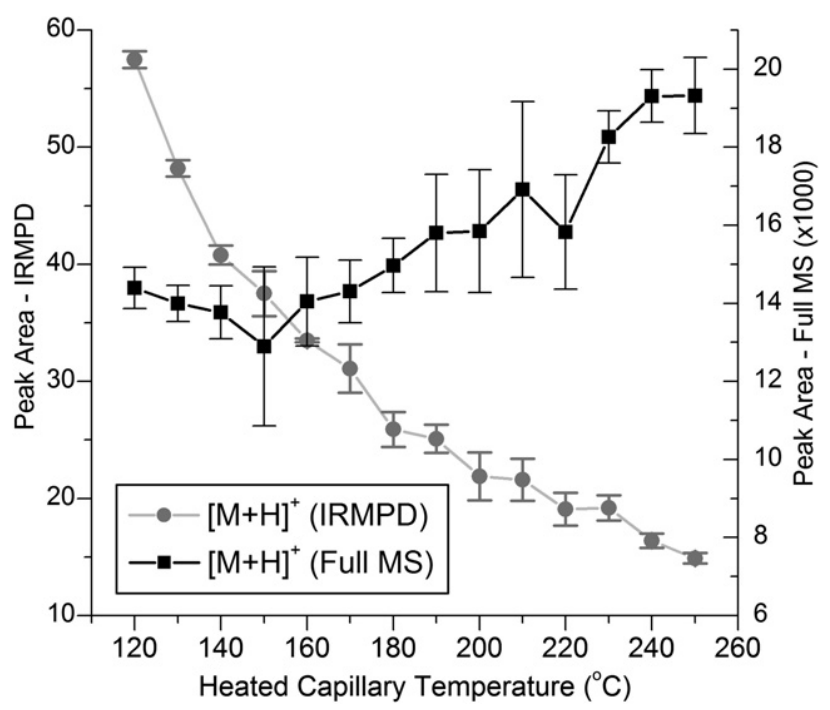

Figure 4. Plot of $[\mathrm{M}+\mathrm{H}]^{+}$peak areas in the ESI mass spectra (black squares, [filled square]) and upon IR irradiation (30 ms, 50 W) of $m / z$ 1900-2000 (gray circles, [filled circle]) as a function of heated inlet capillary temperature when infusing a sample of reserpine ( $1 \mu \mathrm{M}$ in $\mathrm{MeOH})$. Ions of $m / z 1900-2000$ were subjected to IR irradiation and the abundance of the $[\mathrm{M}+\mathrm{H}]^{+}$product ion was calculated. Data were acquired in triplicate; reserpine is represented by $\mathrm{M}$.

protonated, doubly protonated, and sodium-cationized bradykinin (Supplemental Figure 2). As the isolation window increased from $\mathrm{m} / \mathrm{z} 1100-1200$ to $\mathrm{m} / \mathrm{z} 1900-$ 2000 , the abundance of the peptide cations produced upon supplemental IR activation decreased, thus suggesting that the greatest portion of the chemical noise consists of bradykinin ions solvated by only a few solvent molecules with $\mathrm{m} / \mathrm{z}$ values just above that of the protonated peptide, as opposed to large clusters of bradykinin ions surrounded by dozens of solvent molecules resulting in "noise" ions of much higher $\mathrm{m} / \mathrm{z}$. IR and collisional activation of an $\mathrm{m} / \mathrm{z}$ region between that of the $1+$ and $2+$ charge states of fibrinopeptide A produced both the singly and doubly protonated peptide, which suggests that charged solvent clusters also solvate the peptides (see Supplemental Figure 3). Supplemental activation of this noise region also yielded stable hydrated forms of the peptide- up to six water molecule adducts were observed. The efficiency of converting noise into analyte ions, defined as the sum of the peak areas of the bradykinin ions produced by supplemental IR activation divided by the total area of the noise region in the isolation mass spectra, was also measured as a function of the $\mathrm{m} / \mathrm{z}$ isolation and irradiation window (Supplemental Figure 2). Although the total abundance of bradykinin produced upon IR irradiation decreases with higher $\mathrm{m} / \mathrm{z}$ windows, the conversion of chemical noise into bradykinin ions remains relatively constant at $19.8 \% \pm 2.1 \%$ averaged across the $\mathrm{m} / \mathrm{z}$ isolation and irradiation windows, suggesting that at least $\sim 20 \%$ of the chemical noise is composed of solvated analyte ions.
At the high standard operating pressures of QITs, low power IR irradiation does not result in dissociation of covalent bonds of most analyte ions due to a near equivalent rate of ion activation through photon absorption and collisional cooling with the buffer gas [16]. The non-resonant nature of IR activation provides an inherent advantage over collisional activation in that all ions in the trap are simultaneously irradiated by the laser beam and thus effectively desolvated. It has been previously shown that broadband auxiliary waveforms can be used to heat a wider range of ions for successful noise reduction [8], a procedure that is not feasible on the current generation of commercial quadrupole ion traps.

\section{Conclusions}

Chemical noise in ESI mass spectra was reduced by low power supplemental IR activation and produced protonated analyte ions. IR irradiation increased the abundances of protonated peptides and proteins by $30 \%-70 \%$ upon isolation and irradiation of a wide $\mathrm{m} / \mathrm{z}$ range. Regions of chemical noise were drastically reduced upon IR irradiation and produced higher abundances of protonated analyte ions than collisional activation due to the non-resonant activation nature of photoabsorption. We speculate that solvated analyte ions and solvent-analyte clusters constitute a large portion of the chemical noise. Further application of supplemental IR activation in linear QITs before ion ejection or tandem MS could be used to reduce noise and increase analyte ion signal, particularly for LC-MS analyses in which survival of a higher abundance of solvated ions is expected.

\section{Acknowledgments}

The authors gratefully acknowledge funding from the NSH (CHE0718320) and the Welch Foundation (F1155).

\section{Appendix A Supplementary Material}

Supplementary material associated with this article may be found in the online version at doi:10.1016/ j.jasms.2009.08.008.

\section{References}

1. Fenn, J. B.; Mann, M.; Meng, C. K.; Wong, S. F.; Whitehouse, C. M. Electrospray Ionization for Mass Spectrometry of Large Biomolecules. Science 1989, 246, 64-71.

2. Loo, J. A.; Udseth, H. R.; Smith, R. D. Collisional Effects on the Charge Distribution of Ions from Large Molecules, Formed by ElectrosprayIonization Mass Spectrometry. Rapid Commun. Mass Spectrom. 1988, 2, 207-210.

3. Light-Wahl, K. J.; Schwartz, B. L.; Smith, R. D. Observation of the Noncovalent Quaternary Associations of Proteins by Electrospray Ionization Mass Spectrometry. J. Am. Chem. Soc. 1994, 116, 5271-5278.

4. Chowdhury, S. K.; Katta, V.; Chait, B. T. An Electrospray-Ionization Mass Spectrometer with New Features. Rapid Commun. Mass Spectrom. 1990, 4, 81-87. 
5. Aebi, B.; Henion, J. D. The Reduction of Chemical Noise in an Atmospheric Pressure Ionization/Ion Spray Interface for Mass Spectrometry. Rapid Commun. Mass Spectrom. 1996, 10, 947-951.

6. Jarvis, M. J. Y.; Koyanagi, G. K.; Zhao, X.; Covey, T. R.; Bohme, D. K. Scrubbing Ions with Molecules: Kinetic Studies of Chemical Noise Reduction in Mass Spectrometry Using Ion-Molecule Reactions with Dimethyl Disulfide. Anal. Chem. 2007, 79, 4006-4012.

7. Guo, X.; Bruins, A. P.; Covey, T. R.; Troetzmueller, M.; Lankmayr, E. Alternative Reagents for Chemical Noise Reduction in Liquid Chromatography-Mass Spectrometry Using Selective Ion-Molecule Reactions. J. Am. Soc. Mass Spectrom. 2009, 20, 105-111.

8. Ramsey, R. S.; Goeringer, D. E.; McLuckey, S. A. Active Chemical Background and Noise Reduction in Capillary Electrophoresis/IonTrap Mass Spectrometry. Anal. Chem. 1993, 65, 3521-3524.

9. Krutchinsky, A. N.; Chait, B. T. On the Nature of the Chemical Noise in MALDI Mass Spectra. I. Am. Soc. Mass Spectrom. 2002, 13, 129-134.

10. Sachon, E.; Clodic, G.; Blasco, T.; Bolbach, G. Protein Desolvation in UV Matrix-Assisted Laser Desorption/Ionization (MALDI). J. Am. Soc. Mass Spectrom. 2007, 18, 1880-1890.
11. Cech, N. B.; Enke, C. G. Practical Implications of Some Recent Studies in Electrospray Ionization Fundamentals. Mass Spectrom. Rev. 2002, 20, 362-387.

12. Rodriguez-Cruz, S. E.; Klassen, J. S.; Williams, E. R. Hydration of Gas-Phase Gramicidin S $(\mathrm{M}+2 \mathrm{H})^{2+}$ Ions Formed by Electrospray: The Transition from Solution to Gas-Phase Structure. J. Am. Soc. Mass Spectrom. 1997, 8, 565-568.

13. Rodriguez-Cruz, S. E.; Klassen, J. S.; Williams, E. R. Hydration of Gas-Phase Ions Formed by Electrospray Ionization. J. Am. Soc. Mass Spectrom. 1999, 10, 958-968.

14. Gardner, M. W.; Vasicek, L. A.; Shabbir, S.; Anslyn, E. V.; Brodbelt, J. S. Chromogenic Cross-Linker for the Characterization of Protein Structure by Infrared Multiphoton Dissociation Mass Spectrometry. Anal. Chem. 2008, 80, 4807-4819.

15. Gardner, M. W.; Smith, S. I.; Ledvina, A. R.; Madsen, J. A.; Coon, J. J. Schwartz, J. C.; Stafford, G. C., Jr.; Brodbelt, J. S. Infrared Multiphoton Dissociation of Peptide Cations in a Dual Quadrupole Linear Ion Trap Mass Spectrometer. Anal. Chem. in press, doi:10.1021/ac901313.

16. McLuckey, S. A.; Goeringer, D. E. Slow Heating Methods in Tandem Mass Spectrometry. J. Mass Spectrom. 1997, 32, 461-474. 